\title{
HÖRMANDER'S CONDITION AND A CONVOLUTION OPERATOR GENERALIZING RIESZ POTENTIALS
}

\author{
JONG-GUK BAK \\ (Communicated by Andrew Bruckner)
}

\begin{abstract}
Under certain hypotheses including a Hörmander-type condition on the convolution kernel $K$ we show that $K * f$ belongs to the space $\operatorname{BMO}\left(\mathbf{R}^{n}\right)$ whenever $f$ belongs to the space $L^{p, \infty}\left(\mathbf{R}^{n}\right)$ (weak $L^{p}$ ) for certain $p$.
\end{abstract}

Let $1<r<\infty$ and $1 / r+1 / r^{\prime}=1$. Consider a convolution operator $T f=K * f$ on $\mathbf{R}^{n}$ which satisfies the following two conditions:

$$
\|T f\|_{q_{0}} \leq B_{1}\|f\|_{p_{0}}
$$

for some $p_{0}, q_{0}$ with $1 / p_{0}=1 / q_{0}+1 / r^{\prime}, 1<p_{0}<r^{\prime}$, and

$$
\int_{|x|>2|y|}|K(x-y)-K(x)|^{r} d x \leq B_{2} \quad \forall y \neq 0 .
$$

Hörmander $[\mathrm{H}]$ proved that conditions (i) and (ii) imply that $T$ is of weak type $(1, r)$; hence, by interpolation and duality $T$ is of strong type $(p, q)$ for all $p, q$ with $1 / p=1 / q+1 / r^{\prime}, 1<p<r^{\prime}$. (We wish to thank Mr. Jung Soo Rhee for bringing this result to our attention.) A condition like (ii) is often referred to as Hörmander's condition.

Our present observation is that (i) and (ii) also imply the boundedness of $T$ from $L^{r^{\prime}}$ to $\operatorname{BMO}\left(\mathbf{R}^{n}\right)$. If (ii) is replaced by a stronger condition (ii') (see below), we obtain the stronger conclusion that $T$ is bounded from weak $L^{r^{\prime}}$ to $\operatorname{BMO}\left(\mathbf{R}^{n}\right)$. We state and prove our result in the second form. Our proof relies heavily on the method of proof of the boundedness from $L^{\infty}$ to BMO of the Calderón-Zygmund singular integral operator (see [S1, GR]). The symbol $\|\cdot\|_{L^{p, q}}\left(\right.$ or $\left.\|\cdot\|_{p, q}\right)$ denotes the Lorentz space norm (see $\left.[\mathrm{O}, \mathrm{SW}]\right)$, and $\|\cdot\|_{*}$ denotes the $\operatorname{BMO}\left(\mathbf{R}^{n}\right)$ norm (see $[\mathrm{GR}]$ ).

Theorem. Suppose that $T$ satisfies (i) (or merely that $T$ is of restricted weak type $\left.\left(p_{0}, q_{0}\right):\left(\mathrm{i}^{\prime}\right)\|T f\|_{q_{0}, \infty} \leq B_{1}\|f\|_{p_{0}, 1}\right)$ for some $1<r<\infty$. If $K$ satisfies

$$
\|K(x-y)-K(x)\|_{L^{r, 1}\left(E_{y} ; d x\right)} \leq B_{2} \quad \forall y \neq 0,
$$

where $E_{y}=\left\{x \in \mathbf{R}^{n}:|x|>2|y|\right\}$, then there exists a constant $A$ such that

$$
\|T f\|_{*} \leq A\|f\|_{L^{r^{\prime}, \infty}\left(\mathbf{R}^{n}\right)} .
$$

Received by the editors May 8, 1992.

1991 Mathematics Subject Classification. Primary 42B99, 44A35, 26 A33. 
Proof. It is enough to show that there exists a constant $A$ such that for every $f$ and cube $Q$ there is a constant $a_{Q}$ such that

$$
\frac{1}{|Q|} \int_{Q}\left|T f(y)-a_{Q}\right| d y \leq \frac{A}{2}\|f\|_{L^{r^{\prime} \cdot \infty}\left(\mathbf{R}^{n}\right)} .
$$

Let $Q^{*}$ denote the cube with the same center as $Q$, but $2 \sqrt{n}$ times the diameter. By translation invariance we may assume that $Q$ and $Q^{*}$ are centered at the origin. Let $a_{Q}=\int_{\left(Q^{*}\right)^{c}} K(-t) f(t) d t$. Write $f_{1}=f \cdot \chi_{Q^{*}}$ and $f_{2}=f-f_{1}$. Then

$$
T f(y)-a_{Q}=T f_{1}(y)+\int_{\left(Q^{*}\right)^{c}}(K(y-t)-K(-t)) f(t) d t=T f_{1}(y)+J .
$$

By Hölder's inequality for Lorentz spaces (see [O]) and (i) (or (i')),

$$
\begin{aligned}
\frac{1}{|Q|} \int_{Q}\left|T f_{1}(y)\right| d y & \leq|Q|^{-1}\left\|T f_{1}\right\|_{L^{q_{0}, \infty}(Q)}\|1\|_{L^{q_{0}^{\prime}, 1}(Q)} \\
& =C|Q|^{-1 / q_{0}}\left\|T f_{1}\right\|_{L^{q_{0}, \infty}(Q)} \leq C B_{1}|Q|^{-1 / q_{0}}\left\|f_{1}\right\|_{p_{0}, 1} \\
& \leq C B_{1}|Q|^{-1 / q_{0}}\left\|\chi_{Q^{*}}\right\|_{q_{0}, 1}\|f\|_{r^{\prime}, \infty} \leq C B_{1}\|f\|_{r^{\prime}, \infty}
\end{aligned}
$$

Also, by Hölder's inequality and (ii'),

$$
\begin{aligned}
\frac{1}{|Q|} \int_{Q}|J| d y & \leq \frac{1}{|Q|} \int_{y \in Q} \int_{|t|>2|y|}|K(y-t)-K(-t) \| f(t)| d t d y \\
& \leq \frac{1}{|Q|} \int_{Q}\|K(y-t)-K(-t)\|_{L^{r, 1}\left(E_{y} ; d t\right)}\|f\|_{r^{\prime}, \infty} d y \leq B_{2}\|f\|_{r^{\prime}, \infty} .
\end{aligned}
$$

Combining these estimates gives (1) with $A=2\left(C B_{1}+B_{2}\right)$.

Examples. If $K * f$ is the Riesz potential $I_{\alpha}(f)$ of order $\alpha, 0<\alpha<n$, then (i) holds with $r^{\prime}=n / \alpha$ (see [S2]). An easy calculation shows that condition (ii') follows from the estimate $|K(x-y)-K(x)| \leq C|y||x|^{\alpha-n-1},|x|>2|y|$. So it follows that $\left\|I_{\alpha}(f)\right\|_{*} \leq A\|f\|_{L^{n / \alpha, \infty}\left(\mathbf{R}^{n}\right)}$. (The weaker estimate $\left\|I_{\alpha}(f)\right\|_{*} \leq$ $A\|f\|_{L^{n / \alpha}\left(\mathbf{R}^{n}\right)}$ is contained in [A].) An immediate corollary of this result is the estimate $\left\|J_{\alpha}(f)\right\|_{*} \leq A\|f\|_{L^{n / \alpha, \infty}\left(\mathbf{R}^{n}\right)}$ for the Bessel potential $J_{\alpha}(f)$, which was originally proved by Stein and Zygmund [SZ] (see also [S2, p. 164]).

\section{REFERENCES}

[A] D. Adams, A note on Riesz potentials, Duke Math. J. 42 (1975), 765-778.

[GR] J. Garcia-Cuerva and J. L. Rubio de Francia, Weighted norm inequalities and related topics, North-Holland Math. Stud., vol. 116, North-Holland, Amsterdam and New York, 1986.

$[\mathrm{H}] \quad$ L. Hörmander, Estimates for translation invariant operators in $L^{p}$ spaces, Acta Math. 104 (1960), 93-140.

[O] R. O'Neil, Convolution operators and $L(p, q)$ spaces, Duke Math. J. 30 (1963), 129-142.

[S1] E. M. Stein, Singular integrals, harmonic functions, and differentiability properties of functions of several variables, Proc. Sympos. Pure Math., vol. 10, Amer. Math. Soc., Providence, RI, 1967, pp. 316-335.

[S2] _ Singular integrals and differentiability properties of functions, Princeton Univ. Press, Princeton, NJ, 1970. 
[SW] E. M. Stein and G. Weiss, An introduction to harmonic analysis on Euclidean spaces, Princeton Univ. Press, Princeton, NJ, 1971.

[SZ] E. M. Stein and A. Zygmund, Boundedness of translation invariant operators on Hölder and $L^{p}$ spaces, Ann. of Math. (2) 85 (1967), 337-349.

Department of Mathematics, Florida State University, Tallahassee, Florida 32306 E-mail address: bak@math.f su.edu 